\title{
The Ideal Modern Dentist and Dental Specialist Profile
}

\author{
Armando Montesinos* \\ Department of Orthodontics, National University of Mexico, Mexico
}

*Corresponding author: Armando Montesinos, Department of Orthodontics, Division of Postgraduate Studies and Research, Dental Faculty, National University of Mexico, Mexico

\section{Editorial}

Evolution is a fascinating process, it affects all living things, humans began to evolve several millions years ago, Homo sapiens appear in the fourth stage of human evolution, modern humans acquired culture, religion, philosophical, thought, analytical thinking, technological knowledge, science and language. Humankind has faced since the beginning several challenges, from the necessity to obtain food, hazard environmental conditions, dangers and competition with other species around them; therefore, humankind has faced several challenges to preserve; including health issues, illness and disease.

Medicine science evolution includes continuous medical advances in knowledge, changes from medical traditions to modern approaches to disease and illness, prevention, new medical concepts, development of medical technology, clinical applications, diagnostic procedures and treatment protocols. This evolution is applied to common disease, pathogenic mutations and new pathogens of time present and in the future. The medical team has followed this evolution to maintain the health of human beings. Dentistry has evolved as well in close relationship to Medicine from the empiric procedures in the ancient times to the highly trained dentistry specialists' procedures and treatment protocols. This evolution concept was a big step for humankind to prevent, diagnose, treat and/or control disease and illness.

There is a disadvantage in a fast-paced evolution, physicians, dentists and specialists may forget the basics of medical or dental treatment, so many new concepts, discoveries or technology can blind the professionals and they may forget the simple details. Oral health providers must evolve and adapt in a fast-changing world and society without losing focus on basics and ethics. First we need to understand how the world turns in order to mention an ideal oral health provider profile; patients are influenced by social media about trends and topics in which the information is not always correct, but the influence of the communication sources is more powerful than scientific facts. Finding the right information on the World Wide Web is not always easy, there are many false facts in the Web but patients ask "Dr Web" and diagnose, evaluate professional performance or even auto treat themselves in some particular cases when treatment is available on line, self-treatment is a very dangerous choice. Aggressive marketing attacks dentists and dental specialists; dental companies, manufacturers and the pharmaceutical industry try to impose treatment protocols, products and techniques; scientific knowledge and an evidence based dental practice must be applied daily.

The ideal modern dentist and dental specialist should have these qualities:
a. Highly trained
b. In search of Constant Continuing Education and Training
c. Capable of applying traditional and basic concepts in conjunction with new science discoveries, technologies and innovations

d. Apply an Evidence Based Practice

e. Open to new products, materials, drugs, equipment and treatment protocols but not influenced by commercial companies
f. Respectful of Dental Ethics
g. Good communication skills 


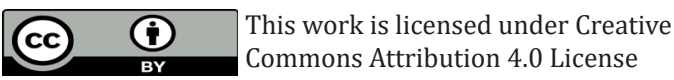

To Submit Your Article Click Here: Submit Article

DOI: 10.32474/MADOHC.2020.04.000185

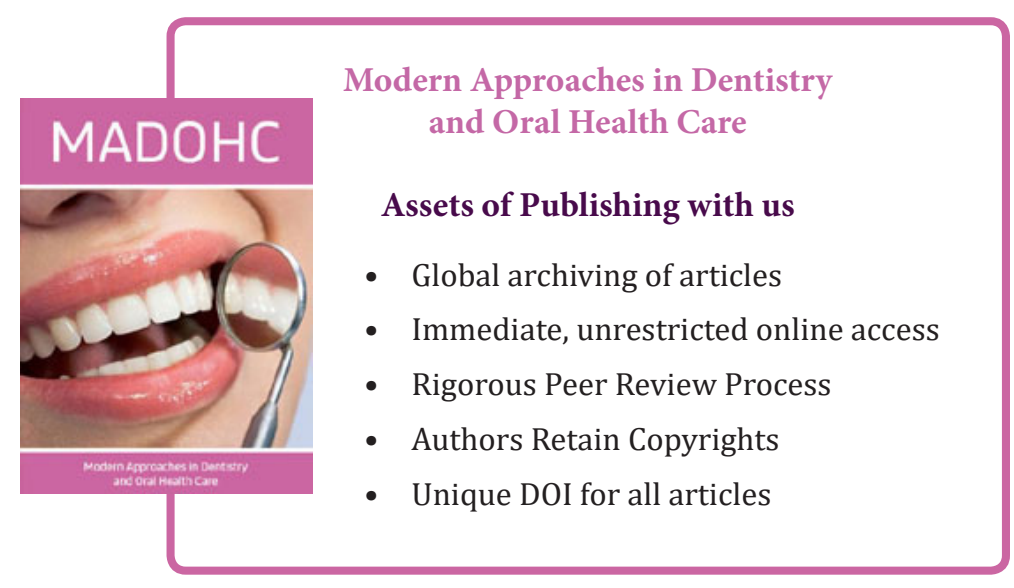

